\title{
Toda Equations and Piecewise Polynomiality for Mixed Double Hurwitz Numbers ${ }^{\star}$
}

\author{
I.P. GOULDEN ${ }^{\dagger}$, Mathieu GUAY-PAQUET ${ }^{\ddagger}$ and Jonathan NOVAK $\S$ \\ $\dagger$ Department of Combinatorics and Optimization, University of Waterloo, \\ 200 University Ave. W., Waterloo, ON, N2L 3G1 Canada \\ E-mail: ipgoulden@uwaterloo.ca \\ URL: https://uwaterloo.ca/math/ian-gouldens-home-page \\ ¥ Département de mathématiques, Université du Québec à Montréal, \\ C.P. 8888, succ. Centre-ville, Montréal, Québec, H3C 3P8 Canada \\ E-mail: mathieu.guaypaquet@lacim.ca \\ $\S$ Department of Mathematics, University of California San Diego, \\ 9500 Gilman Drive, La Jolla, CA 92093-0404 USA \\ E-mail: jinovak@ucsd.edu
}

Received February 02, 2016, in final form April 13, 2016; Published online April 20, 2016

http://dx.doi.org/10.3842/SIGMA.2016.040

\begin{abstract}
This article introduces mixed double Hurwitz numbers, which interpolate combinatorially between the classical double Hurwitz numbers studied by Okounkov and the monotone double Hurwitz numbers introduced recently by Goulden, Guay-Paquet and Novak. Generalizing a result of Okounkov, we prove that a certain generating series for the mixed double Hurwitz numbers solves the 2-Toda hierarchy of partial differential equations. We also prove that the mixed double Hurwitz numbers are piecewise polynomial, thereby generalizing a result of Goulden, Jackson and Vakil.
\end{abstract}

Key words: Hurwitz numbers; Toda lattice

2010 Mathematics Subject Classification: 05A05; 14H70

\section{Introduction}

Consider the right Cayley graph of the symmetric group $S(d)$, as generated by the full conjugacy class of transpositions. This is a $\left(\begin{array}{l}d \\ 2\end{array}\right)$-regular graded graph with levels $L_{0}, L_{1}, \ldots, L_{d-1}$, where $L_{k}$ is the set of permutations with $d-k$ cycles. Each level $L_{k}$ decomposes as the disjoint union of those conjugacy classes in $S(d)$ labelled by Young diagrams with $d-k$ rows.

Let us introduce an edge labelling of the Cayley graph by marking each edge corresponding to the transposition $\tau=(s t)$ with $t$, the larger of the two elements interchanged by $\tau$. This edge labelling was used by Stanley [22] and Biane [1] to study various connections between permutations, parking functions, and noncrossing partitions ${ }^{1}$.

Given integers $k, l \geq 0$ and partitions $\alpha, \beta \vdash d$, let $W^{k, l}(\alpha, \beta)$ denote the number of $(k+l)$-step walks

$$
\sigma=\rho \underbrace{\left(s_{1} t_{1}\right) \cdots\left(s_{k} t_{k}\right)}_{k} \underbrace{\left(s_{k+1} t_{k+1}\right) \cdots\left(s_{k+l} t_{k+l}\right)}_{l}
$$

${ }^{\star}$ This paper is a contribution to the Special Issue on Asymptotics and Universality in Random Matrices, Random Growth Processes, Integrable Systems and Statistical Physics in honor of Percy Deift and Craig Tracy. The full collection is available at http://www.emis.de/journals/SIGMA/Deift-Tracy.html

${ }^{1}$ Stanley and Biane use $s$ as the edge label instead of $t$, but this is a minor difference. 
on the Cayley graph beginning in the conjugacy class $C_{\alpha}$ and ending in the conjugacy class $C_{\beta}$ which satisfy

$$
t_{1} \leq \cdots \leq t_{k}
$$

In words, this monotonicity condition states that the labels of the edges traversed in the first $k$ steps of the walk form a weakly increasing sequence.

While elementary to define, the numbers $W^{k, l}(\alpha, \beta)$ are related to some rather sophisticated mathematics. Let $z, t, u, a_{1}, a_{2}, \ldots, b_{1}, b_{2}, \ldots$ be commuting indeterminates, and form the generating function

$$
\mathbf{W}(z, t, u, A, B)=1+\sum_{d=1}^{\infty} \frac{z^{d}}{d !} \sum_{k, l=0}^{\infty} t^{k} \frac{u^{l}}{l !} \sum_{\alpha, \beta \vdash d} W^{k, l}(\alpha, \beta) p_{\alpha}(A) p_{\beta}(B),
$$

where $p_{\alpha}(A)$ and $p_{\beta}(B)$ denote the power-sum symmetric functions in the variables $A=\left\{a_{1}\right.$, $\left.a_{2}, \ldots\right\}$ and $B=\left\{b_{1}, b_{2}, \ldots\right\}$, respectively. The series

$$
\mathbf{H}(z, t, u, A, B)=\log \mathbf{W}(z, t, u, A, B)
$$

is a well-defined element of $\mathbb{Q}[[z, t, u, A, B]]$. Set

$$
H^{k, l}(\alpha, \beta)=\left[z^{d} t^{k} \frac{u^{l}}{l !} p_{\alpha}(A) p_{\beta}(B)\right] \mathbf{H}(z, t, u, A, B),
$$

where $[X] Y$ denotes the coefficient of the term $X$ in a series $Y$.

The numbers $H^{0, l}(\alpha, \beta)$ were first studied by Okounkov [15], who called them the double Hurwitz numbers. By a classical construction due to Hurwitz - the monodromy construction $H^{0, l}(\alpha, \beta)$ is a weighted count of degree $d$ branched covers of the Riemann sphere by a compact, connected Riemann surface such that the covering map has profile $\alpha$ over $0, \beta$ over $\infty$, and simple ramification over each of the $l$ th roots of unity. The Riemann-Hurwitz formula determines the genus of the covering surface in terms of the ramification data of the covering map:

$$
g=\frac{l+2-\ell(\alpha)-\ell(\beta)}{2} .
$$

Verifying and extending a conjecture of Pandharipande [20] in Gromov-Witten theory, Okounkov proved that the generating function $\mathbf{H}(z, 0, u, A, B)$ is a solution of the 2-Toda hierarchy of Ueno and Takasaki. The 2-Toda hierarchy is a countable collection of partial differential equations, each of which yields a recurrence relation satisfied by the double Hurwitz numbers. A construction of the Toda hierarchy may be found in [17, Section 4]. Kazarian and Lando [12] subsequently showed that, when combined with the ELSV formula [5], Okounkov's result yields a streamlined proof of the Kontsevich-Witten theorem relating intersection theory in moduli spaces of curves to integrable hierarchies.

Goulden, Jackson and Vakil [10] gave an alternative interpretation of the double Hurwitz number $H^{0, l}(\alpha, \beta)$ as counting lattice points in a certain integral polytope. As a consequence of this interpretation and Ehrhart's theorem, it was shown in [10] that, after a simple rescaling, $H^{0, l}(\alpha, \beta)$ is a piecewise polynomial function of the parts of $\alpha$ and $\beta$, when $\ell(\alpha)$ and $\ell(\beta)$ are held fixed. Detailed structural properties of this piecewise polynomial behaviour were postulated in [10], and subsequently shown to hold by Johnson [11] using the combinatorics of the infinite wedge representation of $\mathfrak{g l}(\infty)$. Shadrin, Spitz and Zvonkine [21] have recently generalized double Hurwitz numbers to the setting of the completed cycle theory introduced by Okounkov and Pandharipande [16]. It is shown in [21] that piecewise polynomiality of double Hurwitz numbers with completed cycle insertions follows from a suitable modification of Johnson's arguments. 
The monotone double Hurwitz numbers $H^{k, 0}(\alpha, \beta)$ were introduced by the present authors in [9], where it was shown that they are the combinatorial objects underlying the asymptotic expansion of the Harish-Chandra-Itzykson-Zuber integral, an important special function in random matrix theory. The paper [9] was an outgrowth of the works [13, 14], which developed new connections between unitary matrix integrals, combinatorics, and integrable systems. Structural properties of the monotone single Hurwitz numbers $H^{k, 0}(\alpha)=H^{k, 0}\left(\alpha, 1^{d}\right)$ were studied in detail in $[7,8]$, where it was shown that they enjoy a high degree of structural similarity with the classical single Hurwitz numbers $H^{0, l}(\alpha)=H^{0, l}\left(\alpha, 1^{d}\right)$.

In this article, we extend the theorems of Okounkov and Goulden-Jackson-Vakil to the more general setting of the mixed double Hurwitz numbers $H^{k, l}(\alpha, \beta)$, which interpolate between the classical double Hurwitz numbers $(k=0)$ and the monotone double Hurwitz numbers $(l=0)$.

Theorem 1. The generating function $\mathbf{H}$ is a solution of the 2-Toda hierarchy.

Theorem 2. The mixed double Hurwitz numbers are piecewise polynomial.

\section{Toda equations}

Let us group the transposition generators of $S(d)$ into a matrix,

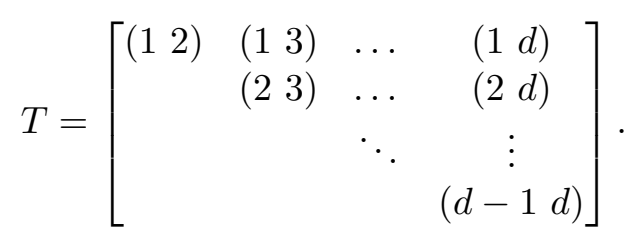

Denote by

$$
J_{2}=\left(\begin{array}{ll}
1 & 2
\end{array}\right), \quad J_{3}=\left(\begin{array}{ll}
1 & 3
\end{array}\right)+\left(\begin{array}{ll}
2 & 3
\end{array}\right), \quad \ldots, \quad J_{d}=\left(\begin{array}{ll}
1 & d
\end{array}\right)+\left(\begin{array}{ll}
2 & d
\end{array}\right)+\cdots+(d-1 d),
$$

the column sums of this matrix, viewed as elements of the group algebra $\mathbb{Q} S(d)$. These elements commute. Set $J_{1}:=0$, and introduce the multiset

$$
\Xi_{d}=\left\{\left\{J_{1}, \ldots, J_{d}, 0,0, \ldots\right\}\right\}
$$

Let $\Lambda$ denote the $\mathbb{Q}$-algebra of symmetric functions. We will consider the evaluation of the complete symmetric function $h_{\mu} \in \Lambda$ indexed by the $(k, l)$-hook Young diagram $\mu=\left(k, 1^{l}\right)$ on the alphabet $\Xi_{d}$. From the definition of the complete symmetric functions, we have

$$
\begin{aligned}
h_{\left(k, 1^{l}\right)}\left(\Xi_{d}\right) & =h_{k}\left(\Xi_{d}\right) h_{1}\left(\Xi_{d}\right)^{l}=\left(\sum_{2 \leq t_{1} \leq \cdots \leq t_{k} \leq d} J_{t_{1}} \cdots J_{t_{k}}\right)\left(\sum_{t=2}^{d} J_{t}\right)^{l} \\
& \left.=\left(\sum_{\substack{2 \leq t_{1} \leq \cdots \leq t_{k} \leq d \\
s_{1}<t_{1}}}\left(s_{1} t_{1}\right)\right) \cdots\left(\sum_{s_{k}<t_{k}}\left(s_{k} t_{k}\right)\right)\right)\left(\sum_{2 \leq t \leq d} \sum_{s<t}(s t)\right)^{l} \\
& =\sum_{\substack{t_{1}, \ldots t_{k+l}=2 \\
t_{1} \leq \cdots \leq t_{k} \\
s_{i}<t_{i}}}^{d}\left(s_{1} t_{1}\right) \cdots\left(s_{k} t_{k}\right)\left(s_{k+1} t_{k+1}\right) \cdots\left(s_{k+l} t_{k+l}\right) .
\end{aligned}
$$

Thus

$$
W^{k, l}(\alpha, \beta)=\left[C_{\left(1^{d}\right)}\right] C_{\alpha} h_{\left(k, 1^{l}\right)}\left(\Xi_{d}\right) C_{\beta},
$$


where we have identified each conjugacy class in $S(d)$ with the formal sum of its elements in $\mathbb{Q} S(d)$. In other words, $W^{k, l}(\alpha, \beta)$ is the normalized character of $C_{\alpha} h_{\left(k, 1^{l}\right)}\left(\Xi_{d}\right) C_{\beta}$ in the regular representation of $\mathbb{Q} S(d)$.

The columns sums of $T$ are known as the Jucys-Murphy elements of $\mathbb{Q} S(d)$. It was observed by Jucys and Murphy (see [3] for a proof) that $e_{r}\left(\Xi_{d}\right)$, the $r$ th elementary symmetric function evaluated on the alphabet of Jucys-Murphy elements, is precisely the sum of the permutations on level $L_{r}$ of the Cayley graph:

$$
e_{r}\left(\Xi_{d}\right)=\sum_{\substack{\mu \vdash d \\ \ell(\mu)=d-r}} C_{\mu} .
$$

In particular, $e_{r}\left(\Xi_{d}\right)$ belongs to the center $Z(d)$ of $\mathbb{Q} S(d)$. Since $\Lambda=\mathbb{Q}\left[e_{1}, e_{2}, \ldots\right]$, the substitution $f \mapsto f\left(\Xi_{d}\right)$ defines a specialization $\Lambda \rightarrow Z(d)$. In fact, since the levels of the Cayley graph generate $Z(d)$, this specialization is surjective [6].

Since $C_{\alpha} h_{\left(k, 1^{l}\right)}\left(\Xi_{d}\right) C_{\beta}$ belongs to the centre of $\mathbb{Q} S(d)$, we can calculate its character in the regular representation using the Fourier transform. Let $\left(V^{\lambda}, \rho^{\lambda}\right), \lambda \vdash d$, be pairwise non-isomorphic irreducible representations of $\mathbb{Q} S(d)$, so that the map

$$
\sigma \mapsto\left(\rho^{\lambda}(\sigma): \lambda \vdash d\right)
$$

defines an algebra isomorphism

$$
\mathbb{Q} S(d) \rightarrow \bigoplus_{\lambda \vdash d} \text { End } V^{\lambda}
$$

The normalized character of $C_{\alpha} h_{\left(k, 1^{l}\right)}\left(\Xi_{d}\right) C_{\beta}$ in the regular representation of $\mathbb{Q} S(d)$ is thus

$$
\begin{aligned}
{\left[C_{\left(1^{d}\right)}\right] C_{\alpha} h_{\left(k, 1^{l}\right)}\left(\Xi_{d}\right) C_{\beta} } & =\sum_{\lambda \vdash d} \operatorname{Tr} \rho^{\lambda}\left(C_{\alpha} h_{\left(k, 1^{l}\right)}\left(\Xi_{d}\right) C_{\beta}\right) \frac{\operatorname{dim} V^{\lambda}}{d !} \\
& =\sum_{\lambda \vdash d} \operatorname{Tr} \rho^{\lambda}\left(C_{\alpha}\right) \rho^{\lambda}\left(h_{\left(k, 1^{l}\right)}\left(\Xi_{d}\right)\right) \rho^{\lambda}\left(C_{\beta}\right) \frac{\operatorname{dim} V^{\lambda}}{d !} \\
& =\sum_{\lambda \vdash d} \operatorname{Tr} \omega^{\lambda}\left(C_{\alpha}\right) \omega^{\lambda}\left(h_{\left(k, 1^{l}\right)}\left(\Xi_{d}\right)\right) \omega^{\lambda}\left(C_{\beta}\right) \frac{\left(\operatorname{dim} V^{\lambda}\right)^{2}}{d !}
\end{aligned}
$$

where, for any $C \in Z(d)$, we denote by $\omega^{\lambda}(C)$ the unique eigenvalue of the scalar operator $\rho^{\lambda}(C) \in$ End $V^{\lambda}$, i.e., $\rho^{\lambda}(C)=\omega^{\lambda}(C) I_{V^{\lambda}}$. The eigenvalue $\omega^{\lambda}(C)$ is known as the central character of $C$ in the representation $\left(V^{\lambda}, \rho^{\lambda}\right)$.

The central character of any conjugacy class $C_{\mu}$ is given, in terms of the usual character

$$
\chi_{\mu}^{\lambda}=\operatorname{Tr} \rho^{\lambda}(\sigma), \quad \sigma \in C_{\mu},
$$

by the formula

$$
\omega^{\lambda}\left(C_{\mu}\right)=\left|C_{\mu}\right| \frac{\chi_{\mu}^{\lambda}}{\operatorname{dim} V^{\lambda}}
$$

The central character of any symmetric function $f$ evaluated on $\Xi_{d}$ is simply

$$
\omega^{\lambda}\left(f\left(\Xi_{d}\right)\right)=f\left(\operatorname{Cont}_{\lambda}\right)
$$

the evaluation of $f$ on the multiset of contents of the Young diagram $\lambda$. This remarkable result is due to Jucys and Murphy, see [3] for a proof. 
Recalling that the Schur functions have the expansion

$$
s_{\lambda}=\sum_{\mu \vdash d} \frac{\left|C_{\mu}\right|}{d !} \chi_{\mu}^{\lambda} p_{\mu},
$$

where $\lambda \vdash d$, the generating function $\mathbf{W}=\mathbf{W}(z, t, u, A, B)$ may be rewritten as follows:

$$
\begin{aligned}
\mathbf{W} & =1+\sum_{d=1}^{\infty} \frac{z^{d}}{d !} \sum_{k, l=0}^{\infty} t^{k} \frac{u^{l}}{l !} \sum_{\alpha, \beta, \lambda \vdash d} W^{k, l}(\alpha, \beta) p_{\alpha}(A) p_{\beta}(B) \\
& =1+\sum_{d=1}^{\infty} \frac{z^{d}}{d !} \sum_{k, l=0}^{\infty} t^{k} \frac{u^{l}}{l !} \sum_{\alpha, \beta, \lambda \vdash d}\left(\frac{1}{d !} \sum_{\lambda \vdash d}\left|C_{\alpha}\right| \chi_{\alpha}^{\lambda} h_{\left(k, 1^{l}\right)}\left(\operatorname{Cont}_{\lambda}\right)\left|C_{\beta}\right| \chi_{\beta}^{\lambda}\right) p_{\alpha}(A) p_{\beta}(B) \\
& =1+\sum_{d=1}^{\infty} z^{d} \sum_{k, l=0}^{\infty} t^{k} \frac{u^{l}}{l !} \sum_{\lambda \vdash d} h_{\left(k, 1^{l}\right)}\left(\operatorname{Cont}_{\lambda}\right)\left(\sum_{\alpha \vdash d} \frac{\left|C_{\alpha}\right|}{d !} \chi_{\alpha}^{\lambda} p_{\alpha}(A)\right)\left(\sum_{\beta \vdash d} \frac{\left|C_{\beta}\right|}{d !} \chi_{\beta}^{\lambda} p_{\beta}(B)\right) \\
& =1+\sum_{d=1}^{\infty} z^{d} \sum_{k, l=0}^{\infty} t^{k} \frac{u^{l}}{l !} \sum_{\lambda \vdash d} h_{\left(k, 1^{l}\right)}\left(\operatorname{Cont}_{\lambda}\right) s_{\lambda}(A) s_{\lambda}(B) \\
& =1+\sum_{d=1}^{\infty} z^{d} \sum_{\lambda \vdash d} s_{\lambda}(A) s_{\lambda}(B)\left(\sum_{k=0}^{\infty} t^{k} h_{k}\left(\operatorname{Cont}_{\lambda}\right)\right)\left(\sum_{l=0}^{\infty} \frac{u^{l}}{l !} h_{1}\left(\operatorname{Cont}_{\lambda}\right)^{l}\right) \\
& =\sum_{\lambda \in \mathcal{Y}} Y(\lambda) s_{\lambda}(A) s_{\lambda}(B) .
\end{aligned}
$$

Here $\mathcal{Y}$ is the set of all Young diagrams (including the empty diagram) and

$$
Y(\lambda)=Y(\lambda ; z, t, u)=\prod_{\square \in \lambda} \frac{z e^{c(\square) u}}{1-c(\square) t},
$$

where for any Young diagram $\lambda$ and cell $\square$ in $\lambda, c(\square)$ denotes the content of this cell, i.e., its column index less its row index. By convention, an empty product equals 1 .

In order to complete the proof of Theorem 1, we appeal to the following result of Orlov and Shcherbin [19], and Carrell [2].

Theorem 3. Let $\left\{y_{k}: k \in \mathbb{Z}\right\}$ be a set of variables indexed by the integers, and set

$$
Y(\lambda)=\prod_{\square \in \lambda} y_{c(\square)}
$$

for each $\lambda \in \mathcal{Y}$. The series

$$
\log \left(\sum_{\lambda \in \mathcal{Y}} Y(\lambda) s_{\lambda}(A) s_{\lambda}(B)\right)
$$

is a solution of the 2-Toda lattice hierarchy in the variables $p_{1}(A), p_{2}(A), \ldots$ and $p_{1}(B)$, $p_{2}(B), \ldots$.

Solutions of the Toda equations of the form described in Theorem 3 are known as diagonal content-product solutions. Our computations above show that the generating function

$$
\mathbf{H}(z, t, u, A, B)=\log \mathbf{W}(z, t, u, A, B)
$$

of the mixed double Hurwitz numbers is a diagonal content-product solution, with

$$
y_{k}=y_{k}(z, t, u)=\frac{z e^{k u}}{1-k t} .
$$


In particular, Okounkov's generating function $\mathbf{H}(z, 0, u, A, B)$ for the classical double Hurwitz numbers is the diagonal content product solution with

$$
y_{k}=z e^{k u},
$$

while the generating function $\mathbf{H}(z, t, 0, A, B)$ for the monotone double Hurwitz numbers is the diagonal content product solution with

$$
y_{k}=\frac{z}{1-k t} .
$$

\section{Piecewise polynomiality}

To prove Theorem 2, let us begin by formulating precisely the relationship between the numbers $W^{k, l}(\alpha, \beta)$ and $H^{k, l}(\alpha, \beta)$. To this end, consider the generating functions $\mathbf{W}$ and $\mathbf{H}$ as elements of $\mathbb{Q}[[t, u, A, B]][[z]]$ by writing

$$
\mathbf{W}(z)=1+\sum_{d=1}^{\infty} \frac{z^{d}}{d !} \mathbf{W}_{d}(t, u, A, B), \quad \mathbf{H}(z)=\sum_{d=1}^{\infty} \frac{z^{d}}{d !} \mathbf{H}_{d}(t, u, A, B),
$$

where, for each $d \geq 1$,

$$
\begin{aligned}
& \mathbf{W}_{d}(t, u, A, B)=\sum_{k, l=0}^{\infty} t^{k} \frac{u^{l}}{l !} \sum_{\alpha, \beta \vdash d} W^{k, l}(\alpha, \beta) p_{\alpha}(A) p_{\beta}(B), \\
& \mathbf{H}_{d}(t, u, A, B)=d ! \sum_{k, l=0}^{\infty} t^{k} \frac{u^{l}}{l !} \sum_{\alpha, \beta \vdash d} H^{k, l}(\alpha, \beta) p_{\alpha}(A) p_{\beta}(B) .
\end{aligned}
$$

Then, by the exponential formula, we have

$$
\mathbf{W}_{d}(t, u, A, B)=\sum_{r=1}^{d} \sum_{P_{1} \sqcup \cdots \sqcup P_{r}} \prod_{j=1}^{r}\left|P_{j}\right| ! \mathbf{H}_{\left|P_{j}\right|}(t, u, A, B)
$$

for each $d \geq 1$, as an identity in $\mathbb{Q}[[t, u, A, B]]$. In this identity, the inner sum is over partitions $P_{1} \sqcup \cdots \sqcup P_{r}$ of $\{1, \ldots, d\}$ into $r$ disjoint nonempty sets. Equating the coefficient of $t^{k} \frac{u^{l}}{l !}$ on either side of this identity, we obtain

$$
\sum_{\alpha, \beta \vdash d} W^{k, l}(\alpha, \beta) p_{\alpha}(A) p_{\beta}(B)=\sum_{r=1}^{d} \sum_{P_{1} \sqcup \cdots \sqcup P_{r}} \prod_{j=1}^{r}\left|P_{j}\right| ! \sum_{\zeta^{j}, \eta^{j} \vdash\left|P_{j}\right|} H^{k, l}\left(\zeta^{j}, \eta^{j}\right) p_{\eta^{j}}(A) p_{\zeta^{j}}(B),
$$

for each $d \geq 1$ and $k, l \geq 0$, as an identity in $\mathbb{Q}[[A, B]]$. Note that the sum on the right hand side depends only on the overall block structure of the set partition $P_{1} \sqcup \cdots \sqcup P_{r}$, and not on the internal structure of the individual blocks. We can thus replace the right hand side by a sum over integer partitions,

$$
\begin{aligned}
\sum_{\alpha, \beta \vdash d} & W^{k, l}(\alpha, \beta) p_{\alpha}(A) p_{\beta}(B) \\
& =\sum_{\theta \vdash d} c_{\theta} \sum_{\substack{\left(\zeta^{1}, \ldots, \zeta^{\ell(\theta)}\right) \\
\zeta^{j} \vdash \theta_{j}}} \sum_{\substack{\left.\eta^{1}, \ldots, \eta^{\ell(\theta)}\right) \\
\eta^{j} \vdash \theta_{j}}}\left(\prod_{j=1}^{\ell(\theta)} H^{k, l}\left(\zeta^{j}, \eta^{j}\right)\right) p_{\zeta^{1} \cup \ldots \cup \zeta^{\ell(\theta)}}(A) p_{\eta^{1} \cup \ldots \cup \eta^{\ell(\theta)}}(B),
\end{aligned}
$$


where the coefficient $c_{\theta}$ is given by

$$
c_{\theta}=\left|f^{-1}(\theta)\right| \prod_{i=1}^{\ell(\theta)} \theta_{i} !
$$

and $f$ denotes the surjection

$$
P_{1} \sqcup \cdots \sqcup P_{r} \mapsto\left(\max _{1 \leq j \leq r}\left|P_{j}\right|, \ldots, \min _{1 \leq j \leq r}\left|P_{j}\right|\right)
$$

from set partitions of $\{1, \ldots, d\}$ onto integer partitions of $d$. The internal sums run over sequences of partitions whose $j$ th element is a partition of the $j$ th part of $\theta$, and if $\left(\mu^{1}, \ldots, \mu^{k}\right)$ is any sequence of partitions then $\mu^{1} \cup \cdots \cup \mu^{k}$ denotes the partition obtained by arranging the parts of $\mu^{1}, \ldots, \mu^{k}$ in weakly decreasing order.

Extracting the coefficient of $p_{\alpha}(A) p_{\beta}(B)$ on each side of the above identity in $\mathbb{Q}[[A, B]]$, we obtain the numerical identity

$$
W^{k, l}(\alpha, \beta)=\sum_{\theta \vdash d} c_{\theta} \sum_{\substack{\left(\zeta^{1}, \ldots, \zeta^{\ell(\theta)}\right) \\ \zeta^{j}+\theta_{j} \\ \zeta^{1} \cup \cdots \cup \zeta^{\ell(\theta)}=\alpha}} \sum_{\substack{\left(\eta^{1}, \ldots, \eta^{\ell(\theta)}\right) \\ \eta^{j} \vdash \theta_{j} \\ \eta^{1} \cup \cdots \cup \eta^{\ell(\theta)}=\beta}} \prod_{j=1}^{\ell(\theta)} H^{k, l}\left(\zeta^{j}, \eta^{j}\right) .
$$

The sum on the right hand side receives non-zero contributions from Young diagrams $\theta$ whose rows can be obtained by gluing together rows of $\alpha$, and by gluing together rows of $\beta$. This is possible if and only if, for each row $\theta_{k}$ of $\theta$, there exist subsets $I_{k}, J_{k} \subseteq\{1, \ldots, d\}$ such that

$$
\sum_{i \in I_{k}} \alpha_{i}=\sum_{j \in J_{k}} \beta_{j}=\theta_{k}
$$

In the case of the one-row Young diagram $\theta=(d)$, the required sets are simply $I_{1}=J_{1}=$ $\{1, \ldots, d\}$, so we have

$$
W^{k, l}(\alpha, \beta)=d ! H^{k, l}(\alpha, \beta)+\cdots,
$$

where the ellipsis stands for contributions from Young diagrams $\theta$ that have at least two rows. These contributions are zero unless the constraint (1) is met for each row of $\theta$. We thus conclude that $W^{k, l}(\alpha, \beta)$ and $H^{k, l}(\alpha, \beta)$ typically agree, up to a factor of $d$ !.

We can give a geometric interpretation of the above as follows. Fix two positive integers $m$ and $n$ and consider the convex region $\mathfrak{R}_{m, n}$ given by

$$
\left\{\left(x_{1}, \ldots, x_{m}, y_{1}, \ldots, y_{n}\right): x_{1} \geq \cdots \geq x_{m}>0, y_{1} \geq \cdots \geq y_{n}>0, \sum_{i=1}^{m} x_{i}=\sum_{j=1}^{n} y_{j}\right\}
$$

in Euclidean space $\mathbb{R}^{m+n}$. Pairs of partitions $(\alpha, \beta)$ such that

$$
|\alpha|=|\beta|, \quad \ell(\alpha)=m, \quad \ell(\beta)=n
$$

may be identified with lattice points in $\mathfrak{R}_{m, n}$ via

$$
(\alpha, \beta) \mapsto\left(\alpha_{1}, \ldots, \alpha_{m}, \beta_{1}, \ldots, \beta_{n}\right) .
$$

Given proper nonempty subsets $I \subset\{1, \ldots, m\}$ and $J \subset\{1, \ldots, n\}$, define a hyperplane $\mathfrak{W}_{I J}$ in $\mathbb{R}^{m+n}$ by

$$
\mathfrak{W}_{I J}=\left\{\left(x_{1}, \ldots, x_{m}, y_{1}, \ldots, y_{n}\right) \in \mathbb{R}^{m+n}: \sum_{i \in I} x_{i}=\sum_{j \in J} y_{j}\right\} .
$$


The hyperplanes $\mathfrak{W}_{I J}$, as $I$ ranges over proper nonempty subsets of $\{1, \ldots, m\}$ and $J$ ranges over proper nonempty subsets of $\{1, \ldots, n\}$, constitute the resonance arrangement of [11]. A chamber $\mathfrak{c}$ of the resonance arrangement is a connected component of the complement of a hyperplane $\mathfrak{W}_{I J}$ in $\mathfrak{R}_{m, n}$. On any chamber $\mathfrak{c}$ of the resonance arrangement, we have that

$$
W^{k, l}(\alpha, \beta)=d ! H^{k, l}(\alpha, \beta)
$$

for all lattice points $(\alpha, \beta) \in \mathfrak{c}$.

Theorem 2 claims that for each chamber $\mathfrak{c}$ there exists a polynomial $p_{\mathfrak{c}}^{k, l}$ in $m+n$ variables such that

$$
H^{k, l}(\alpha, \beta)=p_{\mathfrak{c}}^{k, l}\left(\alpha_{1}, \ldots, \alpha_{m}, \beta_{1}, \ldots, \beta_{n}\right)
$$

for all lattice points $(\alpha, \beta) \in \mathfrak{c}$. From the above discussion and Section 2, we know that $H^{k, l}(\alpha, \beta)$ is given by the character formula

$$
H^{k, l}(\alpha, \beta)=\frac{\left|C_{\alpha}\right|}{d !} \frac{\left|C_{\beta}\right|}{d !} \sum_{\lambda \vdash d} \chi_{\alpha}^{\lambda} h_{\left(k, 1^{l}\right)}\left(\text { Cont }_{\lambda}\right) \chi_{\beta}^{\lambda}, \quad d=|\alpha|=|\beta|,
$$

on any chamber of the resonance arrangement. In order to deduce Theorem 2 from this character formula, we appeal to a recent result of Shadrin, Spitz and Zvonkine [21] which asserts the piecewise polynomiality of a general class of sums of the above form.

Let $\mathbb{C}^{\mathcal{Y}}$ denote the algebra of all functions $\mathcal{Y} \rightarrow \mathbb{C}$. Following Olshanski [18, Proposition 2.4], we define the algebra $\mathbb{A}$ of regular functions on Young diagrams to be the subalgebra of $\mathbb{C}^{\mathcal{Y}}$ generated by the functions

$$
\lambda \mapsto f\left(\mathrm{Cont}_{\lambda}\right), \quad f \in \Lambda,
$$

together with the function $\lambda \mapsto|\lambda|$. Consider the transform

$$
S: \mathbb{C}^{\mathcal{Y}} \rightarrow \mathbb{C}^{\mathcal{Y} \times \mathcal{Y}}
$$

from functions on Young diagrams to functions on pairs of Young diagrams defined by

$$
S^{f}(\alpha, \beta)=\frac{\left|C_{\alpha}\right|}{d !} \frac{\left|C_{\beta}\right|}{d !} \sum_{\lambda \vdash d} \chi_{\alpha}^{\lambda} f(\lambda) \chi_{\beta}^{\lambda},
$$

where $d=|\alpha|=|\beta|$. This definition assumes that $\alpha, \beta$ have the same size; if $|\alpha|>|\beta|$ or vice versa, complete the smaller diagram by adding unicellular rows. As detailed by Olshanski [18], the algebra $\mathbb{A}$ of regular functions on Young diagrams may equivalently be described as the algebra of functions on $\mathcal{Y}$ such that $f(\lambda)$ is a shifted symmetric function of the row lengths $\lambda_{1}, \lambda_{2}, \ldots$ of $\lambda$. Moreover, the shifted power-sums

$$
p_{k}^{*}(\lambda)=\sum_{i \geq 1}\left[\left(\lambda_{i}-i+\frac{1}{2}\right)^{k}-\left(-i+\frac{1}{2}\right)^{k}\right]
$$

generate $\mathbb{A}$ as a polynomial ring, i.e., the functions

$$
p_{\mu}^{*}(\lambda)=\prod_{i=1}^{\ell(\mu)} p_{\mu_{i}}^{*}(\lambda)
$$

form a linear basis of $\mathbb{A}$ as $\mu$ ranges over $\mathcal{Y}$. Building on work of Johnson [11], Shadrin, Spitz, and Zvonkine [21] have used the semi-infinite wedge space formalism to demonstrate that the $S$-transform of $p_{\mu}^{*}$ is piecewise polynomial for each $\mu \in \mathcal{Y}$ (see Theorem 6.3 and Remark 6.5 in [21]). Thus, the piecewise polynomiality of the $S$-transform of an arbitrary regular function is a direct consequence of the results of [21]: 
Theorem 4. Given a regular function $f \in \mathbb{A}$, positive integers $m$ and $n$, and a chamber $\mathfrak{c}$ of the resonance arrangement in $\mathfrak{R}_{m, n}$, there exists a polynomial $p_{\mathfrak{c}}^{f}$ in $m+n$ variables such that

$$
S^{f}(\alpha, \beta)=p_{\mathfrak{c}}^{f}\left(\alpha_{1}, \ldots, \alpha_{m}, \beta_{1}, \ldots, \beta_{n}\right)
$$

for all lattice points $(\alpha, \beta) \in \mathfrak{c}$.

From the character formula (2), we see that Theorem 2 follows by applying Theorem 4 with $f$ the regular function

$$
\lambda \mapsto h_{\left(k, 1^{l}\right)}\left(\operatorname{Cont}_{\lambda}\right) .
$$

Let us close by remarking that structural properties of the polynomials representing mixed double Hurwitz numbers can probably be obtained by applying the methods of [11] and [21]. In view of recent progress in monotone Hurwitz theory [4], this seems to be a very interesting topic for future research.

\section{References}

[1] Biane P., Parking functions of types A and B, Electron. J. Combin. 9 (2002), 7, 5 pages.

[2] Carrell S.R., Diagonal solutions to the 2-Toda hierarchy, Math. Res. Lett. 22 (2015), 439-465, arXiv:1109.1451.

[3] Diaconis P., Greene C., Applications of Murphy's elements, Stanford University Technical Report no. 335, 1989.

[4] Do N., Dyer A., Mathews D.V., Topological recursion and a quantum curve for monotone Hurwitz numbers, arXiv:1408.3992.

[5] Ekedahl T., Lando S., Shapiro M., Vainshtein A., Hurwitz numbers and intersections on moduli spaces of curves, Invent. Math. 146 (2001), 297-327, math.AG/0004096.

[6] Farahat H.K., Higman G., The centres of symmetric group rings, Proc. Roy. Soc. London Ser. A 250 (1959), $212-221$.

[7] Goulden I.P., Guay-Paquet M., Novak J., Monotone Hurwitz numbers in genus zero, Canad. J. Math. 65 (2013), 1020-1042, arXiv:1204.2618.

[8] Goulden I.P., Guay-Paquet M., Novak J., Polynomiality of monotone Hurwitz numbers in higher genera, Adv. Math. 238 (2013), 1-23, arXiv:1210.3415.

[9] Goulden I.P., Guay-Paquet M., Novak J., Monotone Hurwitz numbers and the HCIZ integral, Ann. Math. Blaise Pascal 21 (2014), 71-89, arXiv:1107.1015.

[10] Goulden I.P., Jackson D.M., Vakil R., Towards the geometry of double Hurwitz numbers, Adv. Math. 198 (2005), 43-92, math.AG/0309440.

[11] Johnson P., Double Hurwitz numbers via the infinite wedge, Trans. Amer. Math. Soc. 367 (2015), 64156440, arXiv:1008.3266.

[12] Kazarian M.E., Lando S.K., An algebro-geometric proof of Witten's conjecture, J. Amer. Math. Soc. 20 (2007), 1079-1089, math.AG/0601760.

[13] Matsumoto S., Novak J., Jucys-Murphy elements and unitary matrix integrals, Int. Math. Res. Not. 2013 (2013), 362-397, arXiv:0905.1992.

[14] Novak J., Vicious walkers and random contraction matrices, Int. Math. Res. Not. 2009 (2009), 3310-3327, arXiv:0705.0984.

[15] Okounkov A., Toda equations for Hurwitz numbers, Math. Res. Lett. 7 (2000), 447-453, math.AG/0004128.

[16] Okounkov A., Pandharipande R., Gromov-Witten theory, Hurwitz theory, and completed cycles, Ann. of Math. 163 (2006), 517-560, math.AG/0204305.

[17] Okounkov A., Pandharipande R., The equivariant Gromov-Witten theory of $\mathbf{P}^{1}$, Ann. of Math. 163 (2006), 561-605, math.AG/0207233. 
[18] Olshanski G., Plancherel averages: remarks on a paper by Stanley, Electron. J. Combin. 17 (2010), 43, 16 pages, arXiv:0905.1304.

[19] Orlov A.Yu., Shcherbin D.M., Hypergeometric solutions of soliton equations, Theoret. and Math. Phys. 128 (2001), 906-926.

[20] Pandharipande R., The Toda equations and the Gromov-Witten theory of the Riemann sphere, Lett. Math. Phys. 53 (2000), 59-74, math.AG/9912166.

[21] Shadrin S., Spitz L., Zvonkine D., On double Hurwitz numbers with completed cycles, J. London Math. Soc. 86 (2012), 407-432, arXiv:1103.3120.

[22] Stanley R.P., Parking functions and noncrossing partitions, Electron. J. Combin. 4 (1997), 20, 14 pages. 